\title{
Preliminary process in blast cell morphology identification based on image segmentation methods
}

\author{
Retno Supriyanti ${ }^{1}$, Pangestu F. Wibowo ${ }^{2}$, Fibra R. Firmanda ${ }^{3}$, Yogi Ramadhani ${ }^{4}$, Wahyu Siswandari ${ }^{5}$ \\ 1,2,3,4 Department of Electrical Engineering, Jenderal Soedirman University, Indonesia \\ ${ }^{5}$ Department of Medical, Jenderal Soedirman University, Indonesia
}

\begin{tabular}{|c|c|}
\hline Article Info & ABSTRACT \\
\hline Article history: & \multirow{10}{*}{$\begin{array}{l}\text { The diagnosis of blood disorders in developing countries usually uses } \\
\text { the diagnostic procedure complete blood count (CBC). This is due to } \\
\text { the limitations of existing health facilities so that examinations use standard } \\
\text { microscopes as required in CBC examinations. However, the CBC process } \\
\text { still poses a problem, namely that the procedure for manually counting blood } \\
\text { cells with a microscope requires a lot of energy and time, and is expensive. } \\
\text { This paper will discuss alternative uses of image processing technology in } \\
\text { blast cell identification by using microscope images. In this paper, we will } \\
\text { discuss in detail the morphological measurements which include the diameter, } \\
\text { circumference and area of blast cell cells based on watershed segmentation } \\
\text { methods and active contour. As a basis for further development, we compare } \\
\text { the performance between the uses of both methods. The results show that } \\
\text { the active contour method has an error percentage } 5.15 \% \text { while the watershed } \\
\text { method has an error percentage } 8.25 \% \text {. }\end{array}$} \\
\hline Received Jan 3, 2019 & \\
\hline Revised May 4, 2020 & \\
\hline Accepted May 12, 2020 & \\
\hline Keywords: & \\
\hline Active contour & \\
\hline Blast cell & \\
\hline Developing countries & \\
\hline Image processing & \\
\hline Watershed & \\
\hline
\end{tabular}

Copyright @ 2020 Institute of Advanced Engineering and Science. All rights reserved.

\section{Corresponding Author:}

Retno Supriyanti,

Department of Electrical Engineering,

Jenderal Soedirman University,

Kampus Blater, Jl. Mayjend Sungkono KM 5, Blater, Purbalingga, Central Java.

Email: retno_supriyanti@unsoed.ac.id

\section{INTRODUCTION}

Identification of blood cells is one of the diagnostic procedures used to identify various diseases. This diagnostic procedure is commonly called the $\mathrm{CBC}$. $\mathrm{CBC}$ is a blood test that provides information to doctors about five main parts of blood. The five parts are three types of sell (red blood cells, white blood cells, and platelets) and two types of values (hemoglobin value and hematocrit value) [1]. This CBC procedure is widely applied in developing countries including Indonesia because of limited facilities and resources in the medical field. However, the $\mathrm{CBC}$ process still poses a problem, namely that the procedure for manually counting blood cells with a microscope requires a lot of energy and time, and requires a high cost. Blood cells are classified into several types, namely red blood cells, white blood cells often called as leukocytes, and blood platelets. The blood component has specific roles and functions in the circulation process throughout the body. Blood can experience abnormalities that occur mainly in its constituent structures. This disorder results in a disorder or disease in the body. One blood disorder that can occur is acute lymphoblastic leukemia (ALL).

Cancer death rates including leukemia are higher in developing countries compared to developed countries. This difference reflects differences in risk factors and the success of handling detection, as well as the availability of treatment [2]. According to the latest World Health Organization (WHO) data published in 2017 leukemia deaths in Indonesia reached 9,179 or $0.55 \%$ of total deaths. The age adjusted death rate is 4.20 per 100,000 of population [3]. According to the fact, it is seen that the presence of blood cell abnormalities such as leukemia is a case that needs serious attention. 
As explained in the paragraph above, one of the causes of the high mortality rate of leukemia in developing countries including Indonesia is the limited human resources and health facilities. One alternative to overcome this problem is the implementation of technology that is cheap and easy to use, but also accurate, and one of them is digital image processing. Research on leukemia that involves the use of digital image processing itself is quite a lot. Zhang [3] researched leukocyte detection using digital image processing, specifically a combination of image segmentation and pattern recognition. In the segmentation of the image, he divided the image of leukocytes into several parts. While for the classification he uses support vector machine (SVM). Mohapatra [4] introduced the clustering method using shadowed c-means (SCM) in the segmentation of blood microscopic images. SCM method is used to classify each pixel in 4 clusters. The algorithm is used to separate the nucleus and cytoplasm in each sub-image. Negm [5] in his research, he used a decision support system that included panel selection and segmentation using $\mathrm{K}$ means clustering on some datasets on leukimia identification. Ali [6] proposed an algorithm to isolate and count lymphocytes in the image of white blood cells. The process made includes cell segmentation, scanning algorithms, feature extraction, and lymphocyte cell recognition. Rawat [7], In his research, he proposed a new method for distinguishing acute lymphoblastic leukemia from normal lymphocytes. This method separates leukocytes from other blood cells and then extracts all the information inside. Separation is based on Gray level co-occurrence matrices (GLCM) and form-based features. Prinyakupt [8] he proposed a system for segmenting white blood cells into the area of the nucleus and cytoplasm, extracting appropriate features and classifying them into five types namely basophil, eosinophil, neutrophil, lymphocyte, and monocyte. Liu [9] he proposed a method of calculating red blood cells in full automatic based on hyperspectral microscopic images and combining spatial and spectral information to obtain maximum precision. Lin [10] he proposed a method for classifying five types of leukocytes using a method based on multi-scale regional growth and grouping mean values. The way to do this is to extract the leukocyte texture feature that is visually visible. For the classification, he uses the support vector machine (SVM) method. Li [11] he proposed a method for recognizing leukocytes for human blood smears based on island clustering texture (ICT). The way to do this is to analyse the features of a typical class of leukocytes to form an ICT model. Sarrafzadeh [12] in his research, he used texture features in recognizing leukocytes. He focused on seven categories of texture features in order to get the best category in the classification of leukocytes. Porcu [13] developed a semi-automatic method of extracting all the information on red blood cells and calculating the amount in detail. Umamaheswari [14] proposed an algorithm for segmenting nuclei in white blood cells in leukemia identification. The algorithm developed is based on Otsu's thresholding. Gupta [15] in his research, he presented the PCSeg Tool for segmenting blood plasma cell images. The algorithm that he uses is pre-processing the existing input images by removing all information that is not needed so that all that remains is the region that does provide information about the blood plasma cells only.

Our research also aims to develop a simple system that is efficient and effective in the process of identifying leukocyte cells, according to the limited facilities and infrastructure in several rural areas in Indonesia. Our main contribution is the optimization of the use of simple methods but can provide accurate results in leukocyte identification based on the image of white blood cell smears photographed without illumination settings. We have done some preliminary research [16-21]. Salmam [22] researched facial recognition into a form of emotion using artificial neural networks. Carpio et al., [23] they researched an automatic analysis of bacilli numbers. Also, about calculating the concentration level therein on the sputum sample image of patients with tuberculosis. However, the results obtained are not optimal, because the conditions of the input image are acquired without using the light illumination settings. Therefore it is difficult to apply to input images with various illumination conditions. This paper will discuss the comparison of the use of watershed and active contour methods in obtaining leukocyte segmentation with input images that have random light illumination.

\section{RESEARCH METHOD}

\subsection{Data acquisition}

All the data used in this research came from the Pathology laboratory, Hospital "Dr. Margono Soekardjo," Banyumas, Central Java, Indonesia. Figure 1 shows the stages of the data collection for this research. According to Figure 1, from the pathology laboratory, the medical team examined blood cell smears using a standard microscope. The primary purpose of using this conventional microscope is to create a pilot project so that this can be applied in some rural areas in Indonesia where the district does not yet have a digital microscope facility. Currently, it has only digital microscope facilities, just in large hospitals. In Figure 1, an example of the input image that will be used in this research is also shown, which shows that the image input used has a different illumination level. 


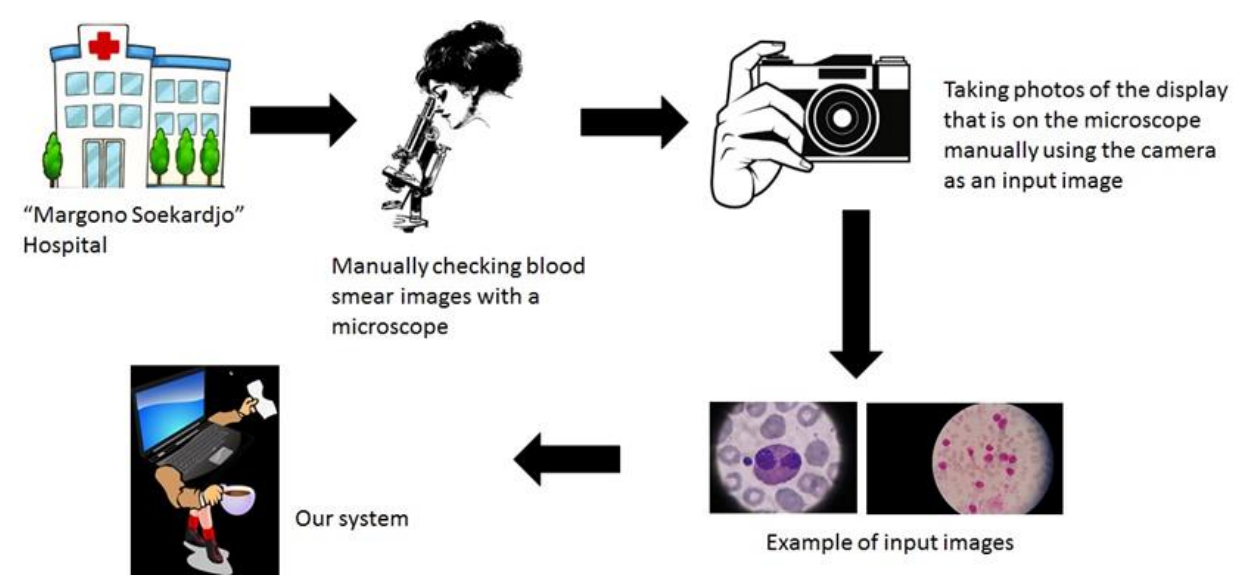

Figure 1. Data acquisition process

\subsection{Pre-processing image}

\subsubsection{Normalization image}

The input images used in this research comes from several types of cameras, so the resolution of each input image is different. According to this case, we set the size of the existing input image so that the calculation results are according to parameters and do not depend on size, but also to speed up data processing time. In this research, the image is resized with a calculated scale using the formula in (1).

$$
R=\frac{400}{S}
$$

In (1), $\mathrm{R}$ states the resize scale, $\mathrm{S}$ is the smallest value of the dimensions of the length and width of the image. The value of 400 is used as the basis of the calculation to determine the scale where the smallest $\mathrm{x}$ or y dimension value is selected as a comparison with the value 400, and the result is used as a scale for the remaining values of $\mathrm{x}$ or $\mathrm{y}$. The above calculation is used so that can adjust the characteristics of images that have different lengths and widths. Moreover, to make it easier to recalculate the original pixel size. Table 1 is a comparison table of original dimensions with resizing dimensions.

Table 1. Comparison of original dimensions and resize dimensions

\begin{tabular}{ccc}
\hline No & Original Dimension & Resize Dimension \\
\hline 1 & $640 \times 480$ & $534 \times 400$ \\
2 & $960 \times 720$ & $534 \times 400$ \\
3 & $4000 \times 3000$ & $534 \times 400$ \\
4 & $4128 \times 2322$ & $712 \times 401$ \\
5 & $581 \times 1032$ & $400 \times 711$ \\
\hline
\end{tabular}

\subsubsection{Cropping}

At this stage, the images obtained are cut using the cropping function in order to obtain the Region of interest (ROI). Cropping is done automatically adjusting the ROI of the image. The process is done by changing the image to binary with a scale of 0.7 then noise cleaning is done by removing objects that have a pixel count of fewer than 4000 pixels. This is done to get objects that only contain ROI. Then scan the object to find the maximum and minimum coordinate values of $\mathrm{X}$ and $\mathrm{Y}$. The results are used to calculate the length and width of the object by subtracting the maximum value of the $\mathrm{x}$ and $\mathrm{y}$ coordinates with the minimum value than using the minimum value of the $\mathrm{x}$ and $\mathrm{y}$ coordinates as the initial coordinate of cropping. Figure 2 shows the image before and after cropping.

\subsubsection{Thresholding}

Thresholding in this system aims to remove other objects other than objects of white blood cells in order to improve accuracy at the stage of segmentation that will be carried out. This step is done by utilizing RGB value differences in white blood cell objects and other objects. Sampling is carried out on five pieces of images that represent the condition of each input image. RGB values are taken from 9 pixels of each image consisting of 3 pixels of white blood cells, 3 pixels of red blood cells, three pieces of background pixelsTable 2 shown this process. 


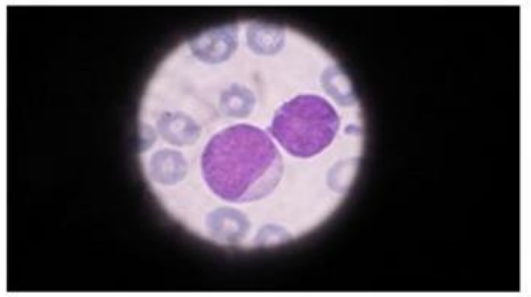

a

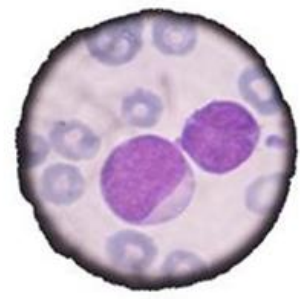

b

Figure 2. (a) Before cropping, (b) after cropping

Table 2. RGB value sampling results

\begin{tabular}{|c|c|c|c|c|}
\hline No & Object & Red & Green & Blue \\
\hline \multirow[t]{9}{*}{$\operatorname{Img} 1$} & \multirow{3}{*}{ White Blood Cell } & 170 & 55 & 86 \\
\hline & & 146 & 40 & 72 \\
\hline & & 170 & 93 & 97 \\
\hline & \multirow[t]{3}{*}{ Red Blood Cell } & 150 & 104 & 88 \\
\hline & & 142 & 101 & 95 \\
\hline & & 127 & 97 & 88 \\
\hline & \multirow[t]{3}{*}{ Background } & 218 & 180 & 109 \\
\hline & & 215 & 182 & 122 \\
\hline & & 11 & 11 & 11 \\
\hline \multirow[t]{9}{*}{$\operatorname{Img} 2$} & \multirow[t]{3}{*}{ White Blood Cell } & 74 & 30 & 125 \\
\hline & & 91 & 37 & 133 \\
\hline & & 131 & 94 & 144 \\
\hline & \multirow[t]{3}{*}{ Red Blood Cell } & 144 & 119 & 149 \\
\hline & & 152 & 129 & 157 \\
\hline & & 153 & 129 & 161 \\
\hline & \multirow[t]{3}{*}{ Background } & 209 & 193 & 204 \\
\hline & & 213 & 196 & 206 \\
\hline & & 13 & 13 & 13 \\
\hline \multirow[t]{9}{*}{$\operatorname{Img} 3$} & \multirow[t]{3}{*}{ White Blood Cell } & 142 & 72 & 144 \\
\hline & & 134 & 67 & 134 \\
\hline & & 144 & 79 & 145 \\
\hline & \multirow[t]{3}{*}{ Red Blood Cell } & 165 & 142 & 268 \\
\hline & & 174 & 157 & 275 \\
\hline & & 179 & 160 & 180 \\
\hline & \multirow[t]{3}{*}{ Background } & 186 & 167 & 187 \\
\hline & & 172 & 158 & 175 \\
\hline & & 12 & 12 & 12 \\
\hline \multirow[t]{9}{*}{$\operatorname{Img} 4$} & \multirow[t]{3}{*}{ White Blood Cell } & 174 & 47 & 118 \\
\hline & & 175 & 48 & 125 \\
\hline & & 174 & 59 & 126 \\
\hline & \multirow[t]{3}{*}{ Red Blood Cell } & 174 & 127 & 137 \\
\hline & & 178 & 131 & 139 \\
\hline & & 181 & 136 & 141 \\
\hline & \multirow[t]{3}{*}{ Background } & 191 & 163 & 159 \\
\hline & & 193 & 163 & 159 \\
\hline & & 0 & 0 & 0 \\
\hline \multirow[t]{9}{*}{$\operatorname{Img} 5$} & \multirow[t]{3}{*}{ White Blood Cell } & 152 & 35 & 105 \\
\hline & & 156 & 40 & 103 \\
\hline & & 138 & 41 & 110 \\
\hline & \multirow[t]{3}{*}{ Red Blood Cell } & 156 & 125 & 138 \\
\hline & & 146 & 113 & 130 \\
\hline & & 164 & 116 & 128 \\
\hline & \multirow[t]{3}{*}{ Background } & 193 & 153 & 153 \\
\hline & & 187 & 152 & 150 \\
\hline & & 1 & 1 & 1 \\
\hline
\end{tabular}

\subsection{Active contour segmentation}

Active contour is a segmentation method using a closed curve model that can move wide or narrow to adjust the boundary of a segmented object [24]. In this experiment, Chan-Vese model contour was used where this model is a region-based active contour. In this model statistical information is used inside or outside the curve to determine the direction of evolution and the amount of energy used as described in (2). 


$$
\begin{aligned}
& \left(c_{1}, c_{2}, C\right)=\mu \text {.length }(C)+v \cdot \operatorname{area}(C) \\
& \quad+\int_{\text {inside }(c)}\left|\mu_{0} \cdot(x, y)-c_{1}\right|^{2} d x d y+\int_{\text {outside }(c)}\left|\mu_{0}(x, y)-c_{2}\right|^{2} d x d y
\end{aligned}
$$

According to (2), the first part defines the length of the curve, the second part of the area in the curve, the third and fourth parts define the difference in intensity of the input image and the average intensity inside and outside the curve. The value of $\mathrm{C}$ is the initial masking curve; the values of $\mathrm{c} 1$ and $\mathrm{c} 2$ are the intensities inside and outside the curve; the value of $\mu 0(\mathrm{x}, \mathrm{y})$ is the input image. The more curves close to the desired object boundary, the smaller the value F. Figure 3 shows an example of using the active contour method in segmenting white blood cell images.

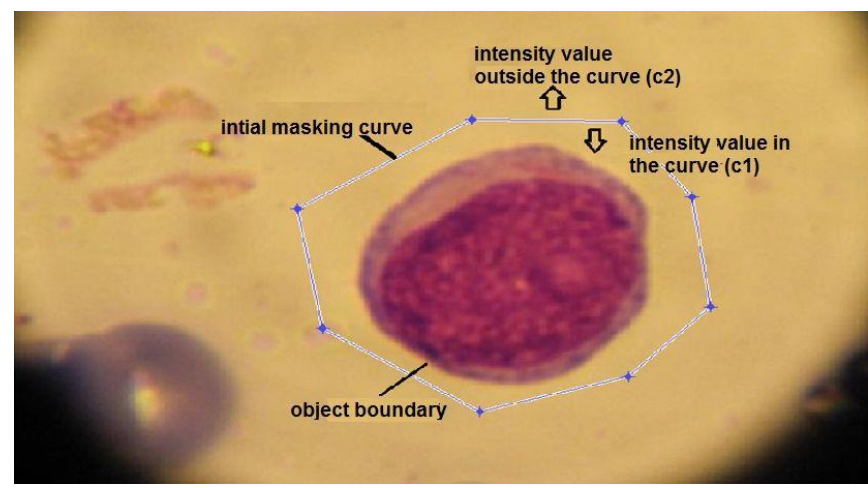

Figure 3. Chan-vese model active contour method

\subsection{Watershed segmentation}

Watershed transformation is a transformation that brings the perspective of two-dimensional imagery in a three-dimensional viewpoint. Watershed transformation works on grayscale images by using watershed transformation, the image as if it has a three-dimensional form, $\mathrm{x}, \mathrm{y}$, and $\mathrm{z}$. The $\mathrm{x}$ and $\mathrm{y}$ fields are the original image itself, while the $\mathrm{z}$ plane is the level of the ash scale in the form of valleys. The thicker the ash level, the deeper the valley [25]. This watershed segmentation method has the advantage of being able to separate objects which are coiled so that objects can be separated as shown in Figure 4. Referring to Figure 4, the object which had previously coincided can be separated by the watershed segmentation method. This is very useful when calculating objects because multicellular cells that are coiled will count as single cells so that the calculation process becomes wrong.
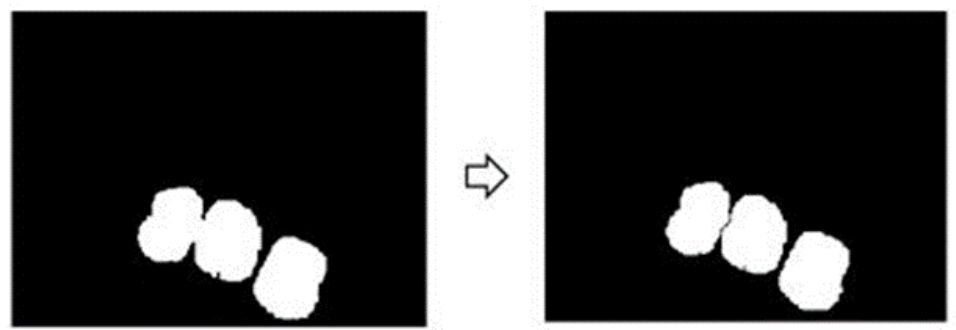

Figure 4. Separation of Objects Coinciding with Watershed Segmentation

\subsection{Post processing}

The post-processing stage is a stage that aims to improve the quality of the second stage image after the segmentation process is carried out. This stage is done because the results of segmentation still allow producing small objects or noise due to the lack of or excessive process of segmentation. Image improvement must be made with good and clean image quality. The operations performed at the post-processing stage are morphological operations, objection elimination on the border edge, elimination of unusual objects, and object names. 


\section{RESULTS AND DISCUSSION}

In this experiment, the variables to be measured in segmentation with the two methods are the detection of the number of leukocytes in each image, area, maximum diameter, minimum diameter and circumference of leukocyte cells. The first experiment was carried out by exploring information using the results of segmentation of the active contour method. Calculation of cell numbers is done by separating cell objects. The process of separating objects is done by calculating the distance transformation on the object to determine the intersection of objects that are overlapped. Distance values are calculated for each nonzero pixel. The model used in this distance transformation is a city block model. The city block model measures distance based on four neighborhoods. Where if the pixel is in area 4, the distance is calculated by one but if it is not then counted two. In order for getting the best result, the distance in the distance transformation needs to be adjusted by removing the small minima locale on the object. Figure 5 shows the results of the separation of objects in this method.

The next process is noise elimination, which functions to eliminate other objects other than the leukocyte cell object. In this process, three parameters are used for three different functions adjusting to the size of the object of the white blood cell. The parameters used are 10000, 3000, and 300 pixels. Then remove the object that is tangent to the edge of the image. Finally, which image is selected is an unusual object and which is not to be removed. The trick is to calculate the degree of roundness of each object with (3).

$$
R=\frac{4 \pi A}{P^{2}}
$$

Referring to (3), then R states the Degree of Roundness, A states Area, and P states Circumference. The roundness used in this experiment is 0.78 so if there is an object with a degree of roundness less than that it will be eliminated. Figure 6 shows some examples of the image results of segmentation by active contour method.

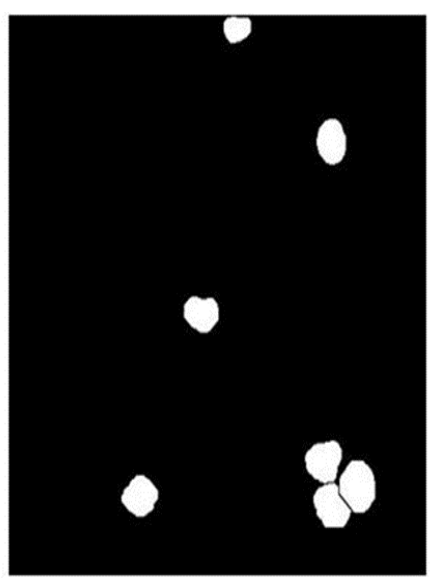

Figure 5. An example of overlapped object separation results

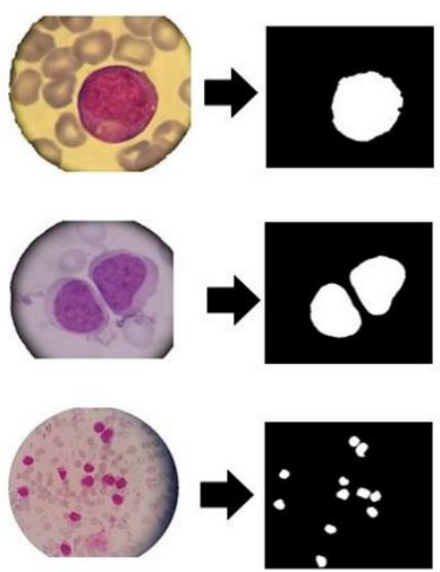

Figure 6. Examples of active contour segmentation result

Referring to Figure 6, the number of leukocyte cells can be segmented using the active contour method. So that based on the segmentation display, the number of leukocyte cells can be calculated automatically. For measurements of diameter, circumference, and area, we refer to (2) above. In (2) above, $\mathrm{C} 1$ and $\mathrm{C} 2$ are two constants which are the average intensity inside and outside the contour, respectively. Moreover, $\mu 0$ is the input image. On active contour without the edge, it will minimize term fitting and add some term regulations, such as the length of the $\mathrm{C}$ curve and the area of the region in $\mathrm{C}$. From the equation, the length of the curve can be measured by (4). While the area is formulated in (5).

$$
\begin{aligned}
& \int_{C} \sqrt{1+\left(y^{\prime}\right)^{2} d x} \\
& L=\frac{1}{2} \int_{C}(x d y-y d x)=\frac{1}{2} \int_{C}\left(x y^{\prime}-y\right) d x
\end{aligned}
$$


In other hand, the watershed segmentation method is a series of methods consisting of watershed transformation, distance transformation, morphological operations, and other related operations. The process of identifying white blood cells in this study is divided into several stages, namely: color threshold, pre-processing, segmentation, post-processing, calculating object parameters. After going through pre-processing as described above, noise is eliminated. Noise removal aims to clean binary images from small objects that are not perfect color thresholds. Also, repairs to the edges of the object are made to be neater and smoother. This operation can be done using image morphology operations. As explained above, watershed segmentation also includes distance transformation. This distance transformation function will produce a set of matrices that contain the transformation value of the distance of each pixel. Distance values are calculated for each nonzero pixel. The chosen model is very decisive towards the results of segmentation because it can allow over segmentation when using the incorrect distance transformation method. According to our experiments using Euclidian, City block, Chess board, Quasy-Euclidian models, and most objects in the form of round objects are not perfect. Therefore, we need a model that has similar shapes or characteristics with the aim that the distance transformation is smooth and later does not cause over-segmentation. With that consideration, it can be seen that the city block method matches the characteristics of the object to be transformed, besides that this model can produce the right segmentation output, while other methods produce over segmentation output.

Referring to (3), properties that can be measured using this function include area, diameter, a circumference of the object. This operation of the elimination of abnormal objects can be done by utilizing the degree of roundness of the object. In order to determine the roundness value of the object, an analysis of the value of the roundness of the object is carried out. Figure 7 shows an example of the property of the degree of roundness of an object. Based on Figure 7, the value of the degree of roundness of strange objects is lower than the standard object. The highest value of the degree of a strange object in the data is 0.53 . Therefore, the degree of roundness is taken at a value of 0.6. Figure 8 is an example of an image that has experienced abnormal object elimination.

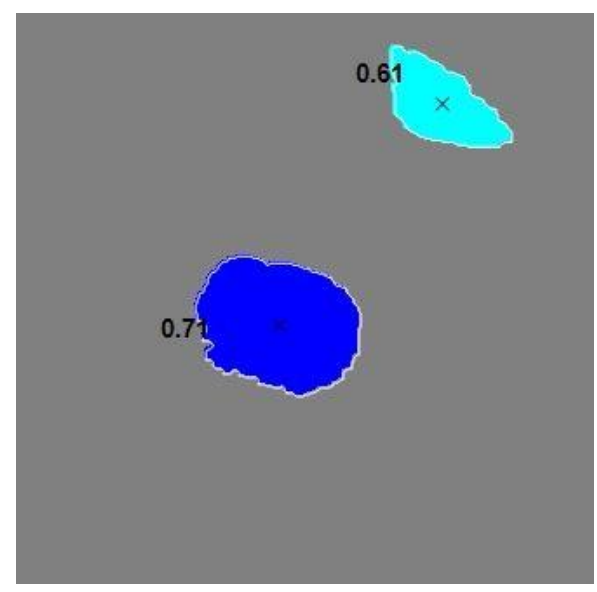

Figure 7. An example of property of roundness object
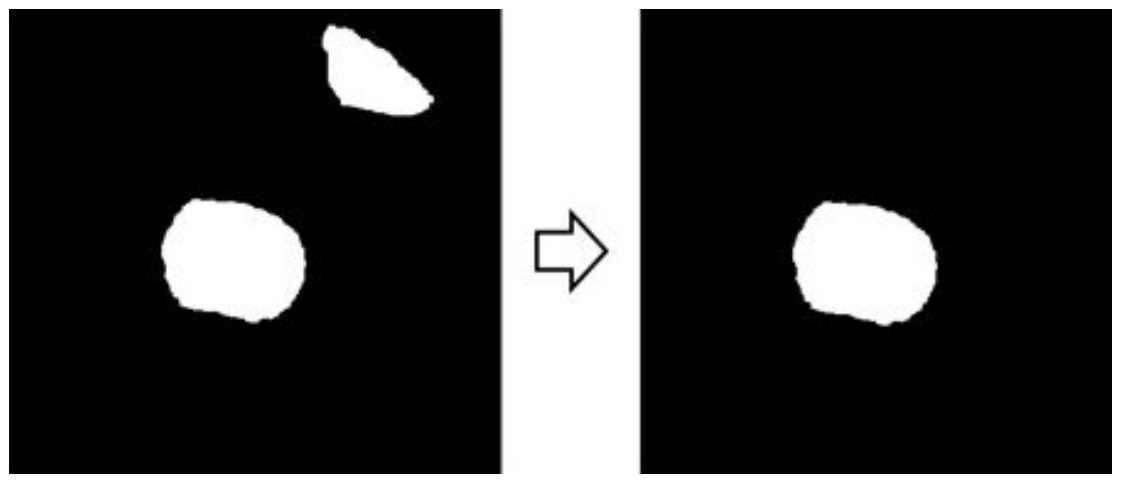

Figure 8. Elimination of abnormal objects 
The way to calculate the circumference of this function is to use eight neighbors; each pixel that does not have neighbors will be counted as a circumference of the object. Calculation of diameter is done by calculating the longest distance and the shortest distance from the edge of the object. The results of diameter properties in this study will get two values for the diameter, namely the maximum diameter and minimum diameter. The extensive calculation is done by counting all pixels on the object. Table 3 shows the results of the calculation of the diameter, circumference and area of leukocytes using the active contour and watershed methods. Referring to Table 3, we make a graph of analysis that shows the relationship between the results obtained in each method as shown in Figure 9.

Referring to Table 3 and Figure 9, in general, the results of the measurement of the two methods give almost the same results for each measurement variable. However, for morphological calculations using watershed segmentation, calculation of properties which include Amount, Diameter, Area, and circumference of leukocyte images. The results of the calculation are in the form of a matrix $n \times 1$, where $n$ is the number of objects detected. The way to calculate the circumference of this function is to use eight neighbors; each pixel that does not have neighbors will be counted as a circumference of the object. Calculation of diameter is done by calculating the longest distance and the shortest distance from the edge of the object. The results of diameter properties in this study will get two values for the diameter, namely the maximum diameter and minimum diameter. The extensive calculation is done by counting all pixels on the object.

Table 3. The results of the calculation of the number, diameter, circumference, and area of leukocytes based on the active contour method

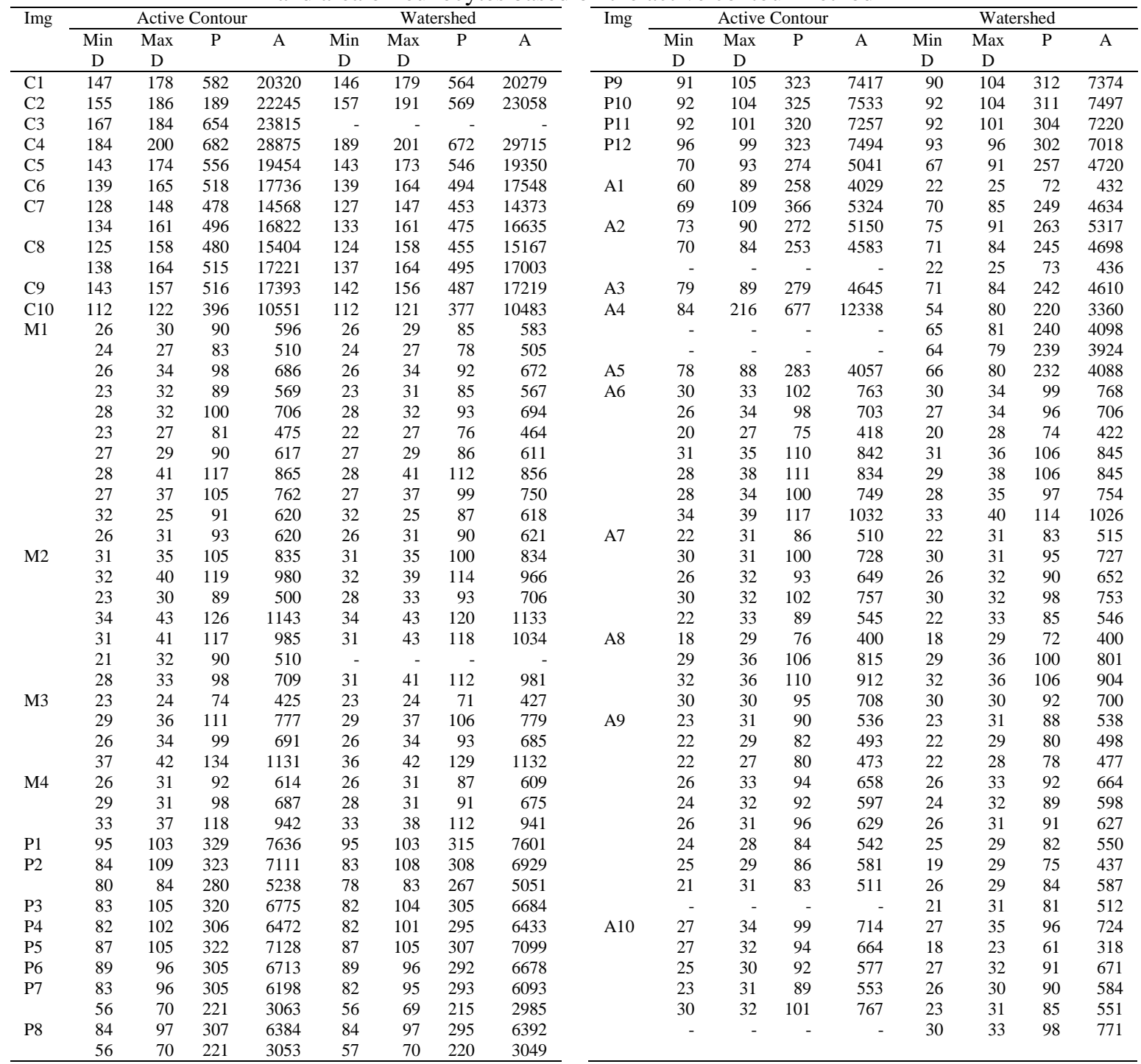




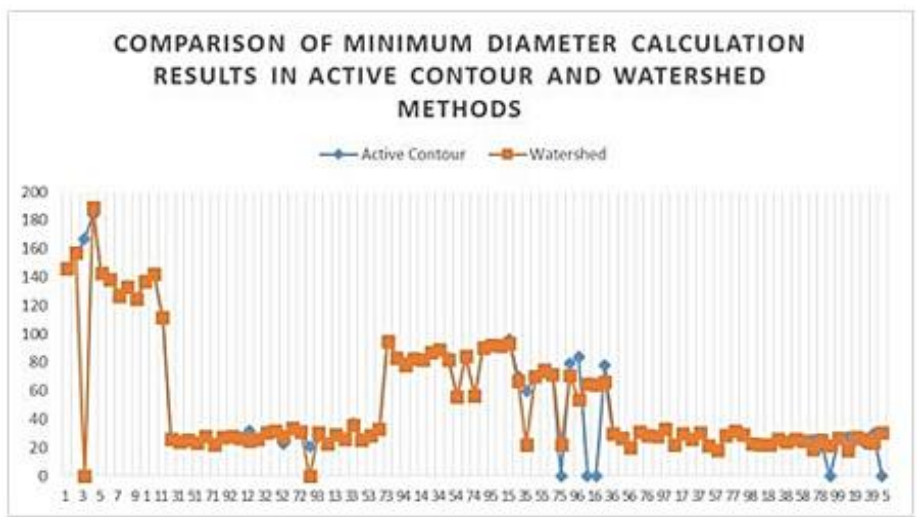

(a)

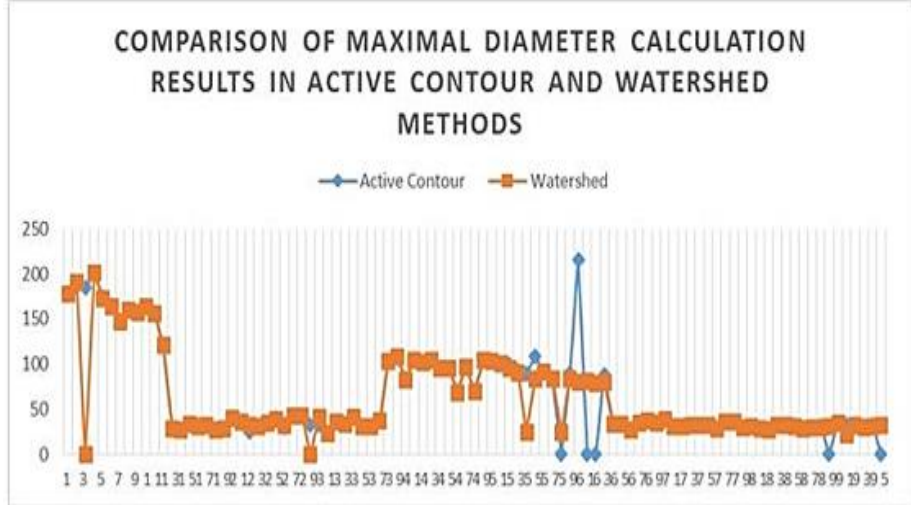

(b)

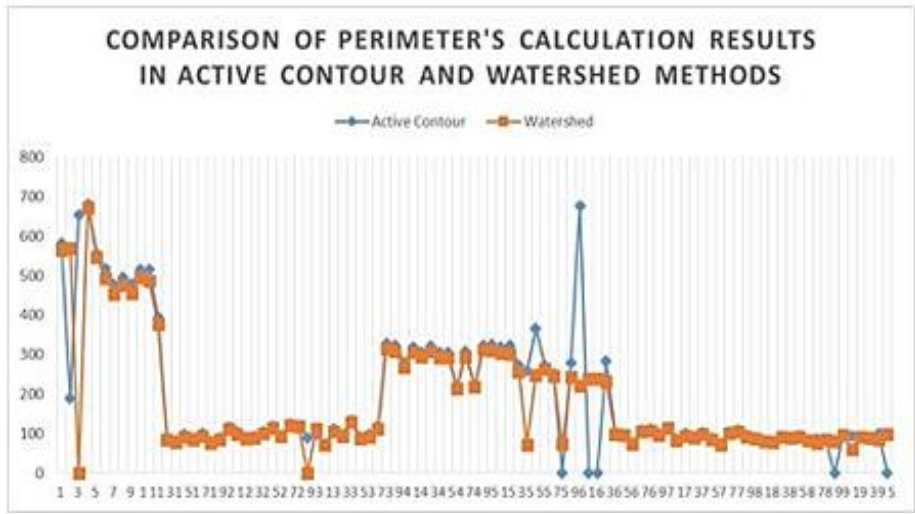

(c)

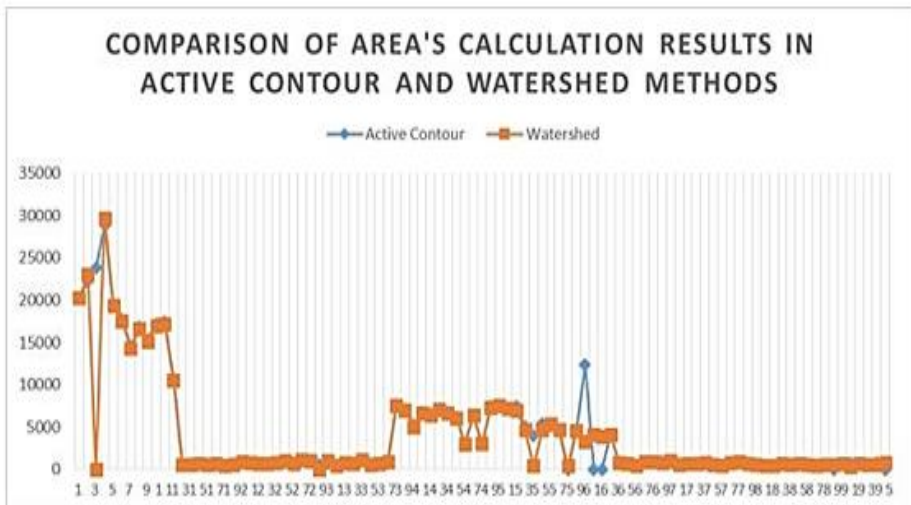

(d)

Figure 9. Graphs of comparison of measurement results based on active contour and watershed methods, (a) minimum diameter, (b) maximal diameter, (c) perimeter, (d) area 
Based on the table above, it can be seen that actual cell count: 97 and total cell deviation calculation: 8 . In order to calculate the percentage of errors can use the following formula as described in (6).

$$
\text { Error percentage }=\left\lceil\frac{\text { Total Deviation }}{\text { Actual Cell Number }}\right\rceil \times 100 \%
$$

Using (6) above, the following results are obtained

$$
\text { Error percentage }=\left|\frac{8}{97}\right| x 100 \%=8.25 \%
$$

Based on the results of watershed segmentation and cell count calculations, it can be concluded that some of the causes of this experiment failure are: (a) Overlapping objects have an irregular shape so that the system does not detect leukocyte cells, so they are not segmented. (b) Other objects besides leukocytes can be detected as leukocytes. This is because the object has characteristics that are similar in color and shape.

In the morphological calculation using the active contour segmentation method, the way to calculate the circumference of this function is to use eight neighbours, each pixel that does not have neighbours will be counted as a circumference of the object. Calculation of diameter is done by calculating the longest distance and the shortest distance from the edge of the object, so in this experiment, there will be two values for the diameter. Extensive calculations are carried out by counting all pixels on the object. In general, the calculation method is the same as the calculation on the watershed segmentation method. We did the morphological calculation using (6) the error percentage can be calculated from morphological calculations based on the segmentation method of active contour.

$$
\text { Error percentage }=\left|\frac{5}{97}\right| \times 100 \%=5.15 \%
$$

Based on the results of segmentation using the active contour method and cell count calculations, it can be concluded that some causes of failure in the experiment are: (a) Overlapped objects have irregular shapes so that over-segmentation occurs or the object is not even segmented. (b) Cells that are truncated or not intact remain detected because they have the size of an object that is almost the same as an intact cell object.

\section{CONCLUSION}

In this experiment, it was found that both for the use of the watershed segmentation method and the active contour segmentation method, for each image that has different characteristics it will require different treatments. Objects other than leukocyte cells can be detected as white blood cells because they have the same intensity of color and shape. Image segmentation using the Watershed Segmentation method has the advantage of being able to separate the blood cells that are huddled together. While for the segmentation of active contour, overlapped object separation can use the distance transformation method. In order to achieve a high percentage of segmentation success, an image that has uniform characteristics and variable control are needed. In this experiment, the calculation based on the active contour method has a lower error percentage than using the watershed segmentation method.

\section{ACKNOWLEDGEMENTS}

We would like to thank the Pathology laboratory, "Prof. Dr. Margono Soekardjo" Hospital for the data that is permitted to be used in this research. The research was funded by the Directorate of Research and Community Service, the Ministry of Research, Technology and Higher Education, Republic of Indonesia through the "Penelitian Terapan" (applied research) scheme.

\section{REFERENCES}

[1] R. Harris, K. Simonsen, and J. Mackay, “Best Tests,” BPAC Organization, New Zealand. [Online]. Available: www.bpac.org.nz, 2013.

[2] D. M. Parkin, F. Bray, J. Ferlay, and P. Pisani, "Global Cancer Statistics, 2002," CA. Cancer J. Clin., vol. 55, no. 2, pp. 74-108, 2009.

[3] W. H. Organization, "World Health Statistics 2017," 2017.

[4] S. Mohapatra, D. Patra, and K. Kumar, "Fast leukocyte image segmentation using shadowed sets," Int. J. Comput. Biol. Drug Des., vol. 5, no. 1, pp. 49-65, 2012.

[5] A. S. Negm, O. A. Hassan, and A. H. Kandil, "A decision support system for Acute Leukaemia classification based on digital microscopic images,” Alexandria Eng. J., vol. 57, no. 4, pp. 2319-2332, 2018. 
[6] A. B. Ali and M. Z. Othman, "Segmentation and Feature Extraction of Lymphocytes WBC using Microscopic Images,” Int. J. Eng. Res. Technol., vol. 3, no. 12, pp. 696-701, 2014.

[7] J. Rawat, A. Singh, H. S. Bhadauria, and J. Virmani, "Computer Aided Diagnostic System for Detection of Leukemia Using Microscopic Images,” Procedia Comput. Sci., vol. 70, pp. 748-756, 2015.

[8] J. Prinyakupt and C. Pluempitiwiriyawej, "Segmentation of white blood cells and comparison of cell morphology by linear and naïve Bayes classifiers," Biomed. Eng. Online, vol. 14, no. 1, pp. 1-19, 2015.

[9] X. Liu, M. Zhou, S. Qiu, L. Sun, H. Liu, Q. Li, and Y. Wang, "Adaptive and automatic red blood cell counting method based on microscopic hyperspectral imaging technology," Journal of Optics, vol. 19, no. 12, 2017. doi: 10.1088/2040-8986/aa95d7

[10] L. Lin and W. Wang, "A robust leukocyte recognition method based on multi-scale regional growth and mean-shift clustering," J. Algorithms Comput. Technol., vol. 12, no. 3, pp. 208-216, 2018.

[11] X. Li and Y. Cao, "A robust automatic leukocyte recognition method based on island-clustering texture," J. Innov. Opt. Health Sci., vol. 09, no. 01, pp. 1650009-1-1650009-13, 2015.

[12] O. Sarrafzadeh, A. M. Dehnavi, Y. Hossein, A. Talebi, and A. Gharibi, "The Best Texture Features for Leukocytes Recognition," J. Med. Signals Sens., vol. 7, no. 4, pp. 220-227 2017.

[13] S. Porcu, A. Loddo, L. Putzu, and C. Di Ruberto, "White Blood Cells Counting Via Vector Field Convolution Nuclei Segmentation," Proceedings of the 13th International Joint Conference on Computer Vision, Imaging and Computer Graphics Theory and Applications, vol. 4, pp. 227-234, 2018.

[14] D. Umamaheswari and S. Geetha, "A Framework for Efficient Recognition and Classification of Acute Lymphoblastic Leukemia with a Novel Customized-KNN Classifier," J. Comput. Inf. Technol., vol. 26, no. 2, pp. 131-140, 2018.

[15] A. Gupta, P. Mallick, O. Sharma, R. Gupta, and R. Duggal, "PCSEG: Color model driven probabilistic multiphase level set based tool for plasma cell segmentation in multiple myeloma," PLoS One, vol. 13, no. 12, pp. 1-22, 2018.

[16] R. Supriyanti, M. M. Afif, I. T. Hasan, Y. Ramadhani, and W. Siswandari, "A Simple Tool for Identifying Outer Shape of White Blood Cell Based on Image Processing Techniques in Order To Develop Health Facilities in Developing Countries," PONTE Int. Sci. Res. J., vol. 73, no. 12, pp. 314-325, 2017.

[17] R. Supriyanti, A. Chrisanty, Y. Ramadhani, and W. Siswandari, "Computer Aided Diagnosis for Screening the Shape and Size of Leukocyte Cell Nucleus based on Morphological Image," International Journal of Electrical and Computer Engineering (IJECE), vol. 8, no. 1, pp. 150-158, 2018.

[18] R. Supriyanti, B. L. Nababan, Y. Ramadhani, and W. Siswandari, "A Simple and Easy-to-Use Tool for Detecting Outer Contour of Leukocytes Based on Image Processing Techniques," 19th International Conference on Biomedical Applications and Bioinformatics, 2017.

[19] R. Supriyanti, G. Satrio, Y. Ramadhani, and W. Siswandari, "Contour Detection of Leukocyte Cell Nucleus Using Morphological Image," J. Phys. Conf. Ser., vol. 824, no. 1, pp. 1-8, 2017.

[20] R. Supriyanti, Rifai. A.rifai, Y. Ramadhani and W. Siswandari, "Influence of camera types in histogram distribution on morphological identification of myeloblast cell based image segmentation," J. Phys. Conf. Ser., vol. 1321, no. 3, pp. 1-6, 2019.

[21] R. Supriyanti, "Technology supporting health services for rural areas based on image processing," J. Phys. Conf. Ser., vol. 1367, pp. 1-12, 2019.

[22] F. Z. Salmam, A. Madani, and M. Kissi, "Emotion recognition from facial expression based on fiducial points detection and using neural network," International Journal of Electrical and Computer Engineering (IJECE), vol. 8, no. 1, pp. 52-59, 2018.

[23] C. del Carpio et al., "An algorithm for detection of Tuberculosis bacilli in Ziehl-Neelsen sputum smear images," International Journal of Electrical and Computer Engineering (IJECE), vol. 9, no. 4, pp. 2968-2981, 2019.

[24] V. Casselles, R. Kimmel, and G. Sapiro, "Geodesic Active Contours," Int. J. Comput. Vis., vol. 22, pp. 61-79, 1997.

[25] R. C. Gonzales and R. E. Woods, "Digital Image Processing," 3rd editio. New Jersey: Prentice Hall, 2008.

\section{BIOGRAPHIES OF AUTHORS}

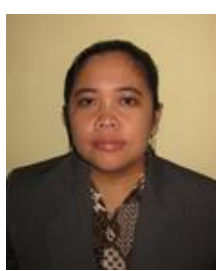

Retno Supriyanti is a Professor at Electrical Engineering Department, Jenderal Soedirman University, Indonesia. She received her PhD in March 2010 from Nara Institute of Science and Technology Japan. Also, she received her M.S degree and Bachelor degree in 2001 and 1998, respectively, from Electrical Engineering Department, Gadjah Mada University Indonesia. Her research interests include image processing, computer vision, pattern recognition, biomedical application, e-health, tele-health and telemedicine.

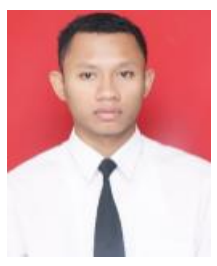

Pangestu Fajar Wibowo received his Bachelor degree from Electrical Engineering Depratment, Jenderal Soedirman University Indonesia. His research interest Image Processing field. 


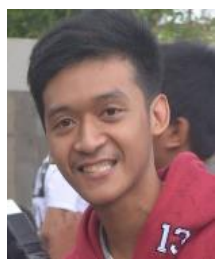

Fibra Rhoma Firmanda received his Bachelor degree from Electrical Engineering Depratment, Jenderal Soedirman University Indonesia. His research interest Image Processing field.

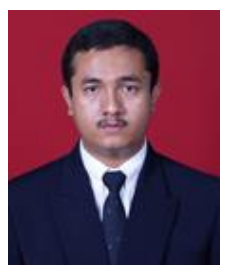

Yogi Ramadhani is an academic staff at Electrical Engineering Department, Jenderal Soedirman University, Indonesia. He received his MS Gadjah Mada Universirt Indonesia, and his Bachelor degree from Jenderal Soedirman University Indonesia. His research interest including Computer Network, Decision Support Syetem, Telemedicine and Medical imaging.

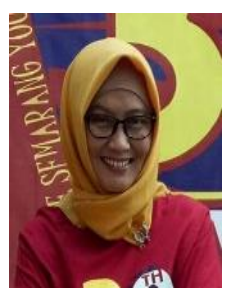

Wahyu Siswandari is an academic staff at Medical Department, Jenderal Soedirman University, Indonesia. She received her Ph.D from Gadjah Mada University. Also, she received his M.S degree and bachelor degree from Diponegoro Indonesia. Her research interest including Pathology, e-health and telemedicine. 